\title{
NUMERICAL COMPUTATION OF SYMMETRY-BREAKING BIFURCATION POINTS
}

\author{
B. S. ATTILI ${ }^{1}$ \\ (Received 14 February 1989; revised 26 February 1991)

\begin{abstract}
We consider symmetry-breaking bifurcation points which arise in parameterdependent nonlinear equations of the form $f(x, \lambda)=0$. These types of bifurcation points are connected to pitchfork bifurcation points. A direct method is used to compute such points. Multiple shooting is used to discretise the two-point boundaryvalue problems to obtain a finite-dimensional problem.
\end{abstract}

\section{Introduction}

Many physical problems can be formulated as a parameter-dependent nonlinear equation of the form

$$
f(x, \lambda)=0,
$$

where $f$ is a $C^{2}$-function which maps $R^{n} \times R$ into $R^{n}$. The point $\left(x_{0}, \lambda_{0}\right)$ is called a regular point of $f(x, \lambda)=0$ if $f_{x}(x, \lambda)$ is nonsingular. It is called a simple singular point of $f(x, \lambda)=0$ if $f_{x}(x, \lambda)$ is singular; $f_{x}^{0}=f_{x}\left(x_{0}, \lambda_{0}\right)$ and $f_{x}^{0 \mathrm{~T}}$ each has one-dimensional null space spanned by $\phi_{0}$ and $\psi_{0}$ respectively. Whether (or not) bifurcation occurs at a singular point depends on the type of singularity, which is described by (in)equalities involving the derivatives of $f(x, \lambda)$ at $\left(x_{0}, \lambda_{0}\right)$. Typically, turning points, and transcritical and pitchfork bifurcation occur.

'K.F.U.P.M., P.O. Box 1927, Dhahran 31261, Saudi Arabia

(C) Australian Mathematical Society, 1993, Serial-fee code 0334-2700/93 
To compute such points, we use the extended system proposed by Griewank and Reddien [4, 5]; that is,

$$
F(x, \lambda)=\left(\begin{array}{c}
f(x, \lambda) \\
g(x, \lambda)
\end{array}\right)=0
$$

where $g(x, \lambda)=u^{\top} f_{x}(x, \lambda) \cdot v$ and $u$ and $v$ are the left and right null vectors of $f_{x}^{0}$ respectively. More on this system will be given in Section 3 (see also Attili $[1,2])$.

The outline of this paper will be as follows. Some assumptions and definitions are given in Section 2. In Section 3, we present the relation between symmetrybreaking bifurcation points and pitchfork bifurcation, and details on the extended system will also be presented. In this section we also present the main theorem of this paper, which shows that a symmetry-breaking bifurcation point is an isolated solution of the extended system. Numerical details are given in the final section.

It is important to note that Werner and Spence [9] have considered computation of such points using a different extended system; also some other investigators considered systems which do not utilise the symmetry, see Moore [6] and Werner [10]. But the system considered here is more direct, and reduces the number of equations and unknowns considerably, in comparison with the system considered by Werner and Spence [9].

\section{Assumptions and definitions}

Before defining what is meant by a symmetry-breaking bifurcation point, we shall recall some standard theory on bifurcation. The point $\left(x_{0}, \lambda_{0}\right)$ is a simple singular point of $f(x, \lambda)=0$ if $f\left(x_{0}, \lambda_{0}\right)=0, N\left(f_{x}^{0}\right)=\operatorname{span}\left\{\phi_{0}\right\}$ and $R\left(f_{x}^{0}\right)=\left\{y \in R^{n}: \psi_{0}^{\top} y=0\right\}$.

DEFINITION 2.1. A simple bifurcation point $\left(x_{0}, \lambda_{0}\right)$ is called a pitchfork bifurcation point of $f(x, \lambda)=0$ if $\psi_{0}^{\top} f_{\lambda}^{0}=0, \psi_{0}^{\top} f_{x x}^{0} \phi_{0} \phi_{0}=0$ and $\psi_{0}^{\top}\left(f_{x \lambda}^{0}+f_{x x}^{0} \phi_{0} v_{0}\right) \neq$ 0 where $f_{x}^{0} v_{0}=-f_{\lambda}^{0}$. If $\psi_{0}^{\top} f_{x x}^{0} \phi_{0} \phi_{0} \neq 0$, the bifurcation point is called transcritical.

We shall assume that (A1): There exists $S \in L\left(R^{n}\right)$, the bounded linear operators mapping $R^{n}$ into $R^{n}$, with $S \neq I$ and $S^{2}=I$ and $f(S x, \lambda)=S f(x, \lambda)$ for all $x \in R^{n}$ and $\lambda \in R^{n}$. 
Here (A1) will give rise to the natural decomposition

$$
R^{n}=R_{s}^{n} \oplus R_{a}^{n}
$$

where

$$
R_{s}^{n}=\left\{x \in R^{n}: S x=x\right\}
$$

is the set of symmetric elements, and

$$
R_{a}^{n}=\left\{x \in R^{n}: S x=-x\right\}
$$

is the set of antisymmetric elements, see Sattinger [7]. The decomposition arises because $x \in R^{n}$ can be written as $2 x=(I+S) x+(I-S) x$, where $S x \in R_{s}^{n}$ and $(I-S) x \in R_{a}^{n}$. To see this relation, note that $S(I+S) x=S x+S^{2} x=$ $S x+I x=(I+S) x$ which implies that $(I+S) x \in R_{s}^{n}$ and also $(I-S) x \in R_{a}^{n}$ since $S(I-S) x=\left(S-S^{2}\right) x=(S-I) x=-(I-S) x$.

DEFINITION 2.2. If $\left(x_{0}, \lambda_{0}\right)$ is a singular point of $f(x, \lambda)=0$, then $\left(x_{0}, \lambda_{0}\right)$ is called a symmetry-breaking bifurcation point of $f(x, \lambda)$ if $x_{0} \in R_{s}^{n}$ and $\phi_{0} \in R_{a}^{n}$ where $\operatorname{span}\left\{\phi_{0}\right\}=N\left(f_{0}^{x}\right)$.

\section{Symmetry breaking and pitchfork bifurcation points}

The singular points in definition (2.2) will be shown to be pitchfork bifurcation points of $f(x, \lambda)=0$ under certain generic conditions. Before doing that, we state some results which can be obtained from assumption (A1).

LEMMA 3.1. Assume that (A1) holds. Then for all $\lambda \in R$ and $x, w, v \in R^{n}$, we have

$$
\begin{aligned}
f_{\lambda}(S x, \lambda) & =S f_{\lambda}(S x, \lambda) \\
f_{x}(S x, \lambda) S v & =S f_{x}(S x, \lambda) v \\
f_{\lambda x}(S x, \lambda) S v & =S f_{\lambda x}(S x, \lambda) v \\
f_{x x}(S x, \lambda) S v S w & =S f_{x x}(S x, \lambda) v w
\end{aligned}
$$

All results in (3.1) can be obtained by differentiating $f(S x, \lambda)=S f(x, \lambda)$ with respect to $\lambda$ or $x$. Using (3.1), one can show that for $\lambda \in R$ and $x \in R_{s}^{n}$

$$
f(x, \lambda), f_{\lambda}(x, \lambda) \in R_{s}^{n} .
$$

This is true if $x \in R_{s}^{n}$ then $S x=x$ and so $f(x, \lambda)=f(S x, \lambda)=S f(x, \lambda)$, which implies that $f(x, \lambda) \in R_{s}^{n}$. The same can be said about $f_{\lambda}(x, \lambda)$. Note 
that $R_{s}^{n}$ and $R_{a}^{n}$ are invariant with regard to $f_{x}(x, \lambda)$ and $f_{\lambda x}(x, \lambda)$. This follows since if $v \in R_{s}^{n}$ then $S v=v$ and so

$$
f_{x}(x, \lambda) v=S f_{x}(x, \lambda) v .
$$

Also, for $v, w \in R_{s}^{n}$ or $v, w \in R_{a}^{n}, f_{x x}(x, \lambda) v w \in R_{s}^{n}$. The reason is that if $v, w$ are in $R_{s}^{n}$, then $S v=v$ and $S w=w$, which yields $f_{x x}(x, \lambda) v w=$ $f_{x x}(x, \lambda) S v w=S f_{x x}(x, \lambda) v w$, and thus $f_{x x}(x, \lambda) v w \in R_{s}^{n}$. If $v \in R_{s}^{n}, w \in R_{a}^{n}$ then $f_{x x}(x, \lambda) v w \in R_{a}^{n}$.

LEMMA 3.2. For every $x \in R_{s}^{n}$, we have $\psi_{0}^{\top} x=0$.

PROOF. Since $R_{s}^{n}$ is invariant with respect to $f_{x}^{0}$ as in (3.3), then $R_{s}^{n}$ is the range of $f_{x}^{0}$. The conclusion now follows from the Fredholm Alternative.

Standard theory of bifurcation shows that at a symmetry-breaking bifurcation point, the solution set of (1.1) will consist of two smooth transversally intersecting branches (see Brezzi, Rappaz and Raviart [3]); that is,

$$
C_{s}=\left\{(x, \lambda): \lambda=\lambda_{0}+\zeta v_{0}+u_{1}(\zeta),|\zeta|<\zeta_{0}\right\}
$$

and

$$
C_{a}=\left\{(x, \lambda): \lambda=\lambda_{0}+o\left(\zeta^{2}\right), x=x_{0}+\zeta \phi_{0}+w_{2}(\zeta),|\zeta|<\zeta_{1}\right\},
$$

where $C_{s}$ and $C_{a}$ refer to the symmetric and antisymmetric branches respectively. The Implicit Function Theorem will guarantee that $C_{s} \subset R_{s}^{n}$ since $f_{x}^{0}$ is restricted to $R_{s}^{n}$ is an isomorphism of $R_{s}^{n}$. Hence $C_{a}$ bifurcates from a symmetric branch of solution $C_{s}$ at a symmetry-breaking bifurcation point. Such branching is given by $\phi_{0} \in R_{a}^{n}$. To compute symmetry-breaking bifurcation points, let us consider the extended system

$$
F(x, \lambda)=\left(\begin{array}{c}
f(x, \lambda) \\
g(x, \lambda)
\end{array}\right)=\left(\begin{array}{c}
f(x, \lambda) \\
-u^{\top} f_{x}(x, \lambda) v
\end{array}\right)=0,
$$

where $u=\psi_{0}$ and $v=\phi_{0}$, the left and right null vectors of $f_{x}^{0}$ respectively, $g(x, \lambda)$ is obtained from the following lemma.

LEMMA 3.3. The systems

$$
\left[\begin{array}{cc}
f_{x} & R \\
T^{q T} & 0
\end{array}\right] \cdot\left[\begin{array}{l}
v \\
g
\end{array}\right]=\left[\begin{array}{l}
0 \\
1
\end{array}\right]
$$


and

$$
\left[\begin{array}{ll}
u^{\top} & g
\end{array}\right] \cdot\left[\begin{array}{cc}
f_{x} & R \\
T^{\top} & 0
\end{array}\right]=\left[\begin{array}{l}
0 \\
1
\end{array}\right]
$$

where $v$ and $u$ are $n \times 1$ vectors, $g$ is a scalar function, $T$ and $R$ are $n \times 1$ vectors, are both uniquely solvable and

$$
\begin{gathered}
g=-u^{\top} f_{x} v \\
g^{\prime}=-u^{\top} f_{x}^{\prime} v+\left(u^{\top} R^{\prime} g+g T^{\prime \top} v\right)
\end{gathered}
$$

where the prime denotes differentiation with respect to $x$ or $\lambda$.

Here $T^{\top}$ and $R$ are normalisations for $v$ and $u$ respectively, which forces $f_{x}$ to be singular, see Attili $[1,2]$ and Griewank and Reddien $[4,5]$. Also $v$ and $R$ are chosen to be in $R_{a}^{n}$. It follows from (3.2) and (3.3) that $F(x, \lambda)$ maps $R_{s}^{n} \times R$ into $R_{s}^{n} \times R$. Hence a symmetry-breaking singular point $\left(x_{0}, \lambda_{0}\right)$ corresponds to a solution $\left(x_{0}, \lambda_{0}\right)$ of (3.4). Moreover, the next theorem shows the relevance of (A1) for pitchfork bifurcation. See Brezzi, Rappaz and Raviart [3].

THEOREM 3.1. Assume that (A1) it holds and let $\left(x_{0}, \lambda_{0}\right)$ be a symmetry breaking simple singular point. Let $F(x, \lambda)$, as in (3.4), be a mapping from $R_{s}^{n} \times R$ into $R_{s}^{n} \times R$ then $\left(x_{0}, \lambda_{0}\right)$ is an isolated solution of (3.4) if and only if $\left(x_{0}, \lambda_{0}\right)$ is a pitchfork bifurcation point.

PROOF. The Jacobian of (3.4) is

$$
\left[\begin{array}{cc}
f_{x} & f_{\lambda} \\
u^{\top} f_{x x} v & u^{\top} f_{x \lambda} v
\end{array}\right]
$$

At $\left(x_{0}, \lambda_{0}\right)$, consider the system

$$
\left[\begin{array}{cc}
f_{x}^{0} & f_{\lambda}^{0} \\
\psi_{0}^{\top} f_{x x}^{0} \phi_{0} & \psi_{0}^{\top} f_{x \lambda} \phi_{0}
\end{array}\right] \cdot\left[\begin{array}{c}
t \\
\alpha
\end{array}\right]=0
$$

which when expanded gives

$$
\begin{array}{cc}
\text { (i) } & f_{x}^{0} \cdot t+\alpha f_{\lambda}^{0}=0 \\
\text { (ii) } \quad \psi_{0}^{\top} f_{x x}^{0} \phi_{0} t+\alpha \psi_{0}^{\top} f_{x \lambda}^{0} \phi_{0}=0 .
\end{array}
$$

Since $-f_{\lambda}^{0}=f_{x}^{0} \cdot v_{0}$ and $f_{x}^{0} \phi_{0}=0$, then from (3.9-i), we have

$$
t=\alpha v_{0}+\beta \phi_{0} .
$$


Substituting (3.10) in (3.9-ii) we get

$$
\alpha \psi_{0}^{\top} f_{x \lambda}^{0} \phi_{0}+\alpha \psi_{0}^{\top} f_{x x}^{0} \phi_{0} v_{0}+\beta \psi_{0}^{\top} f_{x x}^{0} \phi_{0} \phi_{0}=0 .
$$

Moreover $\psi_{0}^{\top} f_{x x}^{0} \phi_{0} \phi_{0}=0$ since $\left(x_{0}, \lambda_{0}\right)$ is a pitchfork bifurcation point, and hence (3.11) becomes

$$
\alpha\left[\psi_{0}^{\top}\left(f_{x \lambda}^{0}+f_{x x}^{0} \phi_{0} v_{0}\right)\right]=0 .
$$

But $\psi_{0}^{\top} f_{x \lambda}^{0} \phi_{0}+f_{x x}^{0} \phi_{0} v_{0} \neq 0$ since $\left(x_{0}, \lambda_{0}\right)$ is a pitchfork bifurcation point, this yields $\alpha=0$. Thus $t=\beta \phi_{0}$ which implies $t \in R_{a}^{n}$, but $t \in R_{s}^{n}$, then $t \in R_{s}^{n} \cap R_{a}^{n}=\{0\}$ and hence (3.10) has only the trivial solution. The solution $(t, \alpha)^{\top}$ is nontrivial if and only if there exist an $\alpha \in R$ and $t \in R_{s}^{n}$ such that

$$
f_{\lambda}^{0}+f_{x}^{0} t=0
$$

and

$$
\psi_{0}^{\top}\left(f_{x \lambda}^{0} \phi_{0}+f_{x x}^{0} \phi_{0} t\right)=0
$$

or equivalently, the null space of (3.8) is trivial if and only if

$$
\psi_{0}^{\top}\left(f_{x \lambda}^{0} \phi_{0}+f_{x x}^{0} \phi_{0} t\right) \neq 0
$$

and $t$ satisfies

$$
f_{\lambda}^{0}+f_{x}^{0} t=0 .
$$

Thus $\left(x_{0}, \lambda_{0}\right)$ is a pitchfork bifurcation point.

The above theorem shows that the symmetry-breaking bifurcation point is an isolated solution of the extended system (3.4), hence it will be used to compute such points. Moreover, the same system can be used to compute certain double singular points $\left(x_{0}, \lambda_{0}\right)$ of $f(x, \lambda)=0$, as we shall show here that $\left(x_{0}, \lambda_{0}\right)$ is again an isolated solution to (3.4), where $F(x, \lambda)=0$ (the extended system) maps $R_{s}^{n} \times R$ into $R_{s}^{n} \times R$. Now assume that

$$
\begin{gathered}
N\left(f_{x}^{0}\right)=\operatorname{span}\left\{\phi_{0} \phi_{1}\right\}, \phi_{0} \in R_{a}^{n}-\{0\} \text { and } \phi_{1} \in R_{s}^{n}-\{0\}, \\
R\left(f_{x}^{0}\right)=\left\{y \in R^{n}: \psi_{i} y=0, i=0,1\right\},
\end{gathered}
$$

and

$$
\psi_{i}^{\top} x=0 \text { for } x \in R_{s}^{n}, \quad \psi_{1}^{\top} x=0 \text { for } x \in R_{a}^{n} .
$$

With these assumptions, we will have the following theorem. 
THEOREM 3.2. Assume that (A1) holds and let $\left(x_{0}, \lambda_{0}\right)$ be a double singular point with $x_{0} \in R_{s}^{n}$. Assume also that (3.12), (3.13) and (3.14) hold. With $F(x, \lambda)$ given by (3.4) considered as a mapping on $R_{s}^{n} \times R$, then $\left(x_{0}, \lambda_{0}\right)$ is an isolated solution of (3.4) if and only if $f_{\lambda}^{0} \notin \operatorname{range}\left(f_{x}^{0}\right)$ and $\psi_{0} f_{x x}^{0} \phi_{0} \phi_{1} \neq 0$.

PRoOF. Consider

$$
\left[\begin{array}{cc}
f_{x}^{0} & f_{\lambda}^{0} \\
\psi_{0}^{\top} f_{x x}^{0} \phi_{0} & \psi_{0}^{\top} f_{x \lambda} \phi_{0}
\end{array}\right] \cdot\left[\begin{array}{c}
t \\
\alpha
\end{array}\right]=0
$$

where the matrix in (3.15) above is the Jacobian of (3.4). Equation (3.15) implies

$$
\text { (ii) } \psi_{0}^{\top} f_{x x}^{0} \phi_{0} t+\alpha \psi_{0}^{\top} f_{x \lambda}^{0} \phi_{0}=0 .
$$

Now since $f_{\lambda}^{0} \notin \operatorname{range}\left(f_{x}^{0}\right)$, then $\psi_{1}^{\top} f_{\lambda}^{0} \neq 0$ and so (3.16-i) implies that $\psi_{1}^{\top} f_{x}^{0} t+\alpha \psi_{1}^{\top} f_{\lambda}^{0}=0$. Further since $\psi_{1}^{\top} f_{x}^{0}=0$, then $\alpha \psi_{1}^{\top} f_{\lambda}^{0}=0$, but $\psi_{1}^{\top} f_{\lambda}^{0} \neq 0$, this shows that $\alpha=0$. Now (3.16-i) becomes $f_{x}^{0} t=0$, and thus $t \in N\left(f_{x}^{0}\right)$, or equivalently $t=a \phi_{0}+b \phi_{1}$. Substituting this in (3.16-ii) we obtain

$$
a \psi_{0}^{\top} f_{x x}^{0} \phi_{0} \phi_{0}+b \psi_{0}^{\top} f_{x x}^{0} \phi_{0} \phi_{1}=0 .
$$

From (3.12), $\phi_{0} \in R_{a}^{n}$ and so $f_{x x}^{0} \phi_{0} \phi_{0} \in R_{s}^{n}$ as shown earlier in this section, also $\psi_{0}^{\top} f_{x x}^{0} \phi_{0} \phi_{0}=0$ since $\psi_{0}^{\top} x=0$ for every $x \in R_{s}^{n}$ (Lemma 3.2), (3.17) becomes $b \psi_{0}^{\top} f_{x x}^{0} \phi_{0} \phi_{1}=0$, which means $b=0$ since $\psi_{0}^{\top} f_{x x}^{0} \phi_{0} \phi_{1} \neq 0$, and so $t=a \phi_{0}$. Here $t=a \phi_{0}$ implies $t \in R_{a}^{n}$ since $\phi_{0} \in R_{a}^{n}$, but $t \in R_{s}^{n}$ since $F(x, \lambda)$ maps $R_{s}^{n} \times R$ into $R_{s}^{n} \times R$, which shows $t \in R_{s}^{n} \cap R_{a}^{n}=\{0\}$ and so $a=0$. Thus (3.15) has only the trivial solution. The other side of the "if and only if" statement follows directly.

A result similar to the above is stated without proof in Werner and Spence [9], where they considered a different extended system than the one presented here.

\section{Numerical examples}

4.1. Coupled cell reaction. Consider the nonlinear operator

$$
f(x, \lambda)=\left[\begin{array}{l}
h\left(x_{1}, x_{2}, \lambda\right) \\
h\left(x_{2}, x_{1}, \lambda\right)
\end{array}\right]
$$


where $x=\left(x_{1}, x_{2}\right) \in R \times R$, which represent a two-cell exothermic reaction (see Werner and Spence [9]), where

$$
h\left(x_{1}, x_{2}, \lambda\right)=x_{1}+\epsilon\left(x_{1}-x_{2}\right)-\lambda \exp \left(x_{1} /\left(1+\zeta x_{1}\right)\right\} .
$$

Here $\epsilon$ is some coupling coefficient and the exponential term is the Arrhenius reaction rate term. Solving the extended system (3.4) with $\epsilon=.01$ and $\zeta=.23$ we obtained $\left(x_{1}, x_{2}, \lambda\right)=(7.7773552,7.7773261, .47827289)$ and $\left(x_{1}, x_{2}, \lambda\right)=(2.430591,2.4306056, .51124817)$, which are two simple symmetry-breaking bifurcation points. With $\epsilon=0$, the simple symmetrybreaking bifurcation coalesces with the simple turning point. This means that assumption (A1) will split the double turning point referred to in Theorem 3.2 into a simple turning point and a simple symmetry-breaking bifurcation point.

4.2. The Duffing equation. The system (3.4) in the previous section will be a basis for the computation of symmetry breaking; that is, pitchfork bifurcation points. Such points will be isolated solutions of (3.4). For more on the experimental aspects, consider the Duffing equation which exhibits secondary bifurcation, see Seydel [8]; that is,

$$
\ddot{x}+2 x^{3}=\lambda \cos t, \quad x(0)=x(2 \pi) .
$$

We are seeking $2 \pi$-periodic solutions. The assumption (A1) of Section 2 is satisfied directly for (4.1) because of the symmetry properties of the cosine for $S \in\left(S_{\alpha}, S_{\beta}, S_{\gamma}\right)$, where

$$
\begin{aligned}
& \left(S_{\alpha} x\right)(t)=x(-t), \\
& \left(S_{\beta} x\right)(t)=-x(\pi-t), \\
& \left(S_{\gamma} x\right)(t)=-x(t-\pi) .
\end{aligned}
$$

Equation (4.1) has a branch of $2 \pi$-periodic functions which are symmetric with respect to all symmetries. On this branch there are several symmetrybreaking bifurcation points at which two symmetries are broken and one symmetry is preserved. See Werner and Spence [9].

We shall discretise (4.1) using the shooting method to obtain a finite-dimensional problem. We start by dividing the interval $[0,2 \pi]$ into four equal subintervals. Let $x(0)=s_{1}, \dot{x}(0)=s_{2}, x(\pi)=s_{3}$ and $\dot{x}(\pi)=s_{4}$ be initial guesses at 0 and $\pi$. With $s_{1}$ and $s_{2}$ as initial guesses, solve the two initial-value problems on $[0, \pi / 2]$ and backward on $[3 \pi / 2,2 \pi]$ to obtain $x_{1}, \dot{x}_{1}$ at $\pi / 2$ and $x_{4}, \dot{x}_{4}$ at $3 \pi / 2$ respectively. Also with $s_{3}$ and $s_{4}$ as initial guesses, solve the two initial-value 
problems backward on $[\pi / 2, \pi]$ and forward on $[\pi, 3 \pi / 2]$ to obtain $x_{2}, \dot{x}_{2}$ at $\pi / 2$ and $x_{3}, \dot{x}_{3}$ at $3 \pi / 2$ respectively. Now

$$
F(s, \lambda)=\left[\begin{array}{c}
x_{1}-x_{2} \\
\dot{x}_{1}-\dot{x}_{2} \\
x_{3}-x_{4} \\
\dot{x}_{3}-\dot{x}_{4}
\end{array}\right] .
$$

For the finite-dimensional problem, $\left(S_{\alpha} x\right)(t)=-x(t)$ will be equivalent to saying $s_{2}=0$ and $s_{4}=0$, and so an analogous symmetry to $S_{\alpha}$ is

$$
S_{1}=\left[\begin{array}{cccc}
1 & 0 & 0 & 0 \\
0 & -1 & 0 & 0 \\
0 & 0 & 1 & 0 \\
0 & 0 & 0 & -1
\end{array}\right]
$$

The second symmetry $\left(S_{\beta} x\right)(t)=-x(\pi-t)$ will be analogous to

$$
S_{2}=\left[\begin{array}{cccc}
0 & 0 & -1 & 0 \\
0 & 0 & 0 & 1 \\
-1 & 0 & 0 & 0 \\
0 & 1 & 0 & 0
\end{array}\right]
$$

Also, $\left(S_{\gamma} x\right)(t)=-x(t-\pi)$ is analogous to

$$
S_{3}=\left[\begin{array}{cccc}
0 & 0 & -1 & 0 \\
0 & 0 & 0 & -1 \\
-1 & 0 & 0 & 0 \\
0 & -1 & 0 & 0
\end{array}\right]
$$

To show that assumption (A1) holds for the finite-dimensional problem, we shall deal with $\tilde{F}(s, \lambda)$, where

$$
\tilde{F}(s, \lambda)=Q F(s, \lambda)
$$

and

$$
Q=\left[\begin{array}{cccc}
1 & -1 & 1 & 1 \\
1 & 1 & 1 & -1 \\
-1 & 1 & 1 & 1 \\
1 & 1 & -1 & 1
\end{array}\right]
$$


Note that solving $F(s, \lambda)=0$ is equivalent to solving $\tilde{F}(s, \lambda)=0$, since $Q$ is invertible. The function $\tilde{F}$ is given by

$$
\tilde{F}(s, \lambda)=\left[\begin{array}{c}
x_{1}-x_{2}+x_{3}-x_{4}-\dot{x}_{1}+\dot{x}_{2}+\dot{x}_{3}-\dot{x}_{4} \\
x_{1}-x_{2}+x_{3}-x_{4}+\dot{x}_{1}-\dot{x}_{2}-\dot{x}_{3}+\dot{x}_{4} \\
-x_{1}+x_{2}+x_{3}-x_{4}+\dot{x}_{1}-\dot{x}_{2}+\dot{x}_{3}-\dot{x}_{4} \\
x_{1}-x_{2}-x_{3}+x_{4}+\dot{x}_{1}-\dot{x}_{2}+\dot{x}_{3}-\dot{x}_{4}
\end{array}\right] .
$$

Now, under the $S_{1}$ symmetry, we have

$$
S_{1} s=S_{1}\left(\begin{array}{c}
s_{1} \\
s_{2} \\
s_{3} \\
s_{4}
\end{array}\right)=\left(\begin{array}{c}
s_{1} \\
-s_{2} \\
s_{3} \\
-s_{4}
\end{array}\right)
$$

Solving the initial-value problems with $s_{1},-s_{2}$ and $s_{3},-s_{4}$, we obtain $x_{4},-\dot{x}_{4}$ on $[0, \pi / 2], x_{3},-\dot{x}_{3}$ on $[\pi, \pi / 2], x_{2},-\dot{x}_{2}$ on $[\pi, 3 \pi / 2]$ and $x_{1},-\dot{x}_{1}$ on $[2 \pi, 3 \pi / 2]$. Using these values we find $\tilde{F}\left(S_{1} s, \lambda\right)$ is the same as $\tilde{F}(s, \lambda)$ with the second component and the fourth component reversed in sign, which is $S_{1} \tilde{F}(s, \lambda)$, and so

$$
\tilde{F}\left(S_{1} s, \lambda\right)=S_{1} \tilde{F}(s, \lambda) .
$$

Similarly, one can prove the same result for the other symmetries, which shows that assumption (A1) is satisfied.

For the computations, we can find the symmetric solution by solving

$$
\ddot{x}_{1}+2 x_{1}^{3}=\lambda \cos t, \quad x_{1}(0)=s_{1}, \quad \dot{x}_{1}(0)=0,
$$

on $[0, \pi / 2]$. In order to find the antisymmetric solution, we solve

$$
\ddot{x}_{1}+2 x_{1}^{3}=\lambda \cos t, \quad x_{1}(0)=0, \quad \dot{x}_{1}(0)=s_{1},
$$

on $[0, \pi / 2]$. There will be no need to solve the other initial-value problems on the other subintervals. The solutions may be extended based on the symmetries. We transform (4.10) and (4.11) into a first-order differential equation to obtain

$$
\begin{aligned}
& \dot{x}_{1}=x_{2}, \\
& \dot{x}_{2}=-2 x_{1}^{3}+\lambda \cos t,
\end{aligned}
$$

together with the initial conditions $x_{1}(0)=s_{1}$ and $x_{2}(0)=0$ in the symmetric case and $x_{1}(0)=0$ and $x_{2}(0)=s_{1}$ in the antisymmetric case. In the symmetric case, we need to satisfy $x_{1}(\pi / 2)=0$ and so

$$
F\left(s_{1}, \lambda\right)=x_{1}(\pi / 2)=0 .
$$


To find $g$ we used $R=1$ and $T^{\top}=1$. With this choice, $g=\partial F / \partial s_{1}$. The function $\partial F / \partial s_{1}$ can be obtained by solving the initial-value problem

$$
\dot{v}_{1}=v_{2}, \quad \dot{v}_{2}=-6 u_{1}^{2} v_{1}, \quad v_{1}(0)=0, \quad v_{2}(0)=1
$$

and $\partial F / \partial \lambda$ is obtained by solving

$$
\dot{w}_{1}=w_{2}, \quad \dot{w}_{2}=\cos t-6 u_{1}^{2} w_{1}, \quad w_{1}(0)=0, \quad w_{2}(0)=0 .
$$

Using Newton's method, $(\underset{g}{F})=0$ was solved with $s_{1}=2.88$ and $\lambda=11$ as initial guesses. We find $s_{1}=2.97410$ and $\lambda=12.0776$. The value of $g=-.111768-10$.

The same system was solved using other initial guesses, $s_{1}=1.2$ and $\lambda=$ 1.35. We find $s_{1}=1.33150$ and $\lambda=1.57408$. Note that this problem was solved by Werner and Spence [9] using finite differences, and similar results were obtained.

\section{Acknowledgement}

The author would like to thank the referees for their valuable comments.

\section{References}

[1] B. Attili, "Multiple shooting and the calculation of some types of singularities in B.V.P's", Int. J. Comp. Math. 32 (1990) 97-111.

[2] B. Attili, "A direct method for the characterization and computation of bifurcation points with corank 2", Computing 48 (1992) 149-159.

[3] F. Brezzi, J. Rappaz and P. Raviart, "Finite dimensional approximation of nonlinear problems, Part III, Simple bifurcation points", Numer. Math. 38 (1981) 1-30.

[4] A. Griewank and G. Reddien, "The approximation of generalized turning points by projection methods with super convergence to the critical parameter", Num. Math. 14 (1975) 354-366.

[5] A. Griewank and G. Reddien, "Characterization and computation of generalized turning points", SIAM J. Numer. Anal. 21 (1984) 186-196.

[6] G. Moore, "The numerical treatment of nontrivial bifurcation points", Num. Funct. Anal. Optim. 2 (1980) 441-472.

[7] D. Sattinger, "Group theoretic methods in bifurcation theory", in Lecture Notes in Math. 762, (1979).

[8] R. Seydel, "Branch switching in bifurcation problems for ordinary differential equation", Num. Math. 41 (1983) 93-116.

[9] B. Werner and A. Spence, "The computation of symmetry breaking bifurcation points", SIAM J. Num. Anal. 21 (1984) 388-399.

[10] H. Werner, "On the numerical approximation of secondary bifurcation problems", in Lecture Notes in Math. 878, (1981), 407-425. 